\title{
Atomic-resolution Operando and Time-resolved In Situ TEM Imaging of Oxygen Transfer Reactions Catalyzed by $\mathrm{CeO}_{2}$-supported Pt Nanoparticles
}

\author{
Joshua Vincent and Peter Crozier
}

Arizona State University, Tempe, Arizona, United States

Oxygen transfer is a critical functionality in automotive exhaust control and clean energy conversion. In these applications, catalytically active metal nanoparticles (e.g., Pt) are often dispersed on reducible oxide supports (e.g., $\mathrm{CeO}_{2}$ ), since reducible oxides can transfer their lattice oxygen to adsorbates at the metal-support interface, i.e., the three-phase boundary [1,2]. Attaining an atomic-level understanding of the catalyticallydriven oxygen transfer process is of great interest to the scientific community for its impact on designing more active oxygen transfer catalysts. This work employs aberration-corrected environmental transmission electron microscopy (ETEM) to investigate the atomic-scale structural dynamics that occur at the $\mathrm{Pt} / \mathrm{CeO}_{2}$ three-phase boundary under reaction conditions (i.e., in situ) and during catalysis (i.e., operando).

Nanostructured $\mathrm{CeO}_{2}$ was synthesized, loaded with 17 wt. \% Pt nanoparticles (NPs), and used as model catalyst systems for $\mathrm{CO}$ oxidation $\left(\mathrm{CO}+1 / 2 \mathrm{O}_{2}\right.$ to $\left.\mathrm{CO}_{2}\right)$. An image-corrected FEI Titan ETEM at $300 \mathrm{kV}$ was used to visualize the atomic structures that form under reaction conditions. For in situ experiments, a high-frame rate direct electron detector was coupled to the ETEM, which enabled transient dynamic behavior to be observed with a temporal resolution of 25 milliseconds $(\mathrm{ms})$. Operando TEM experiments were done by published methods that incorporate modified specimen preparation techniques and in situ electron energy-loss spectroscopy (EELS) to generate, detect, and measure the product gas composition during catalysis [3]. In this way, it is possible to correlate atomic-level observations of active site dynamics with quantitative determinations of catalytic turnover frequency (TOF). Gas pressures and temperatures used in the experiments are provided in the figures. Electron fluxes were kept at $\leq 5,000 \mathrm{e} / \AA^{2} / \mathrm{s}$ to reduce undesirable radiation damage to the specimen and gas.

Figure 1 shows time-averaged, 25-frame operando TEM images of Pt NPs on a (111) surface of a $\mathrm{CeO}_{2}$ support in a reactant atmosphere of 0.57 Torr of slightly-lean $\mathrm{CO}$ and $\mathrm{O}_{2}$. The right side of the figure displays the in situ conversions of $\mathrm{CO}$ and TOFs determined by in situ EELS; a background subtracted spectrum showing the conversion of $\mathrm{CO}$ to $\mathrm{CO}_{2}$ is provided in the figure inset. The time-averaged images show that as the TOF for $\mathrm{CO}$ oxidation increases, catalytically-driven oxygen transfer disrupts the bonds that anchor the $\approx$ $2 \mathrm{~nm}$ Pt NPs, leading to dynamic reconfigurations of the entire nanoparticles and their translational motion $\approx$ $2-5 \AA$ across the support. Interestingly, proximal Ce surface structures up to $\approx 1 \mathrm{~nm}$ away from the $\mathrm{Pt} / \mathrm{CeO}_{2}$ interface undergo concurrent dynamics, evidenced by their blurring in the images. Recent work on $\mathrm{CeO}_{2}$ has linked Ce atom column dynamics to oxygen exchange chemistry [4]. Our findings from operando TEM suggest catalytic enhancements could be attained by engineering the support structure in the vicinity of the interface. An in situ experiment with $25 \mathrm{~ms}$ temporal resolution was performed to further probe the dynamics, and the results are shown in Figure 2. Here a Pt NP on a $\mathrm{CeO}_{2}$ (111) surface was observed to be stable in 0.5 mTorr of inert $\mathrm{N}_{2}$ while the same particle was observed to dynamically restructure in a $10 \mathrm{mTorr}$ atmosphere of $\mathrm{CO}$ and $\mathrm{O}_{2}$, evidenced by a loss of fringe visibility (left side of Figure 2). Interestingly, an examination of the time-series in $\mathrm{CO}$ and $\mathrm{O}_{2}$ with $100 \mathrm{~ms}$ time resolution shows the Pt undergoes a sequence of structural reconfigurations that can be correlated with dynamics at interfacial Ce sites. A shear plane seen at the surface of the $\mathrm{CeO}_{2}$ in reactants but not in $\mathrm{N}_{2}$ suggests the behavior may be driven by oxygen vacancy dynamics from oxygen transfer at the $\mathrm{Pt} / \mathrm{CeO}_{2}$ interface $[5,6]$. 

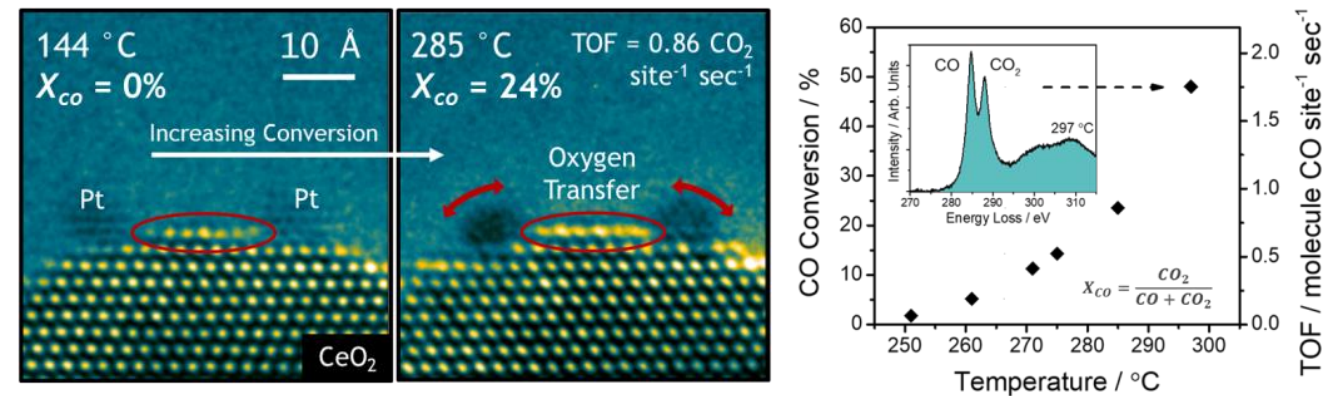

Figure 1. Time-averaged operando images acquired during $\mathrm{CO}$ oxidation (left) and temperature dependent conversion of CO measured in the ETEM (right). The turnover of CO to $\mathrm{CO}_{2}$ is seen to correlate with dynamic rearrangements of the Pt NPs. Furthermore, the surface Ce sites near the interface blur with increasing TOF, pointing to their role in the catalytically-driven oxygen transfer mechanism.
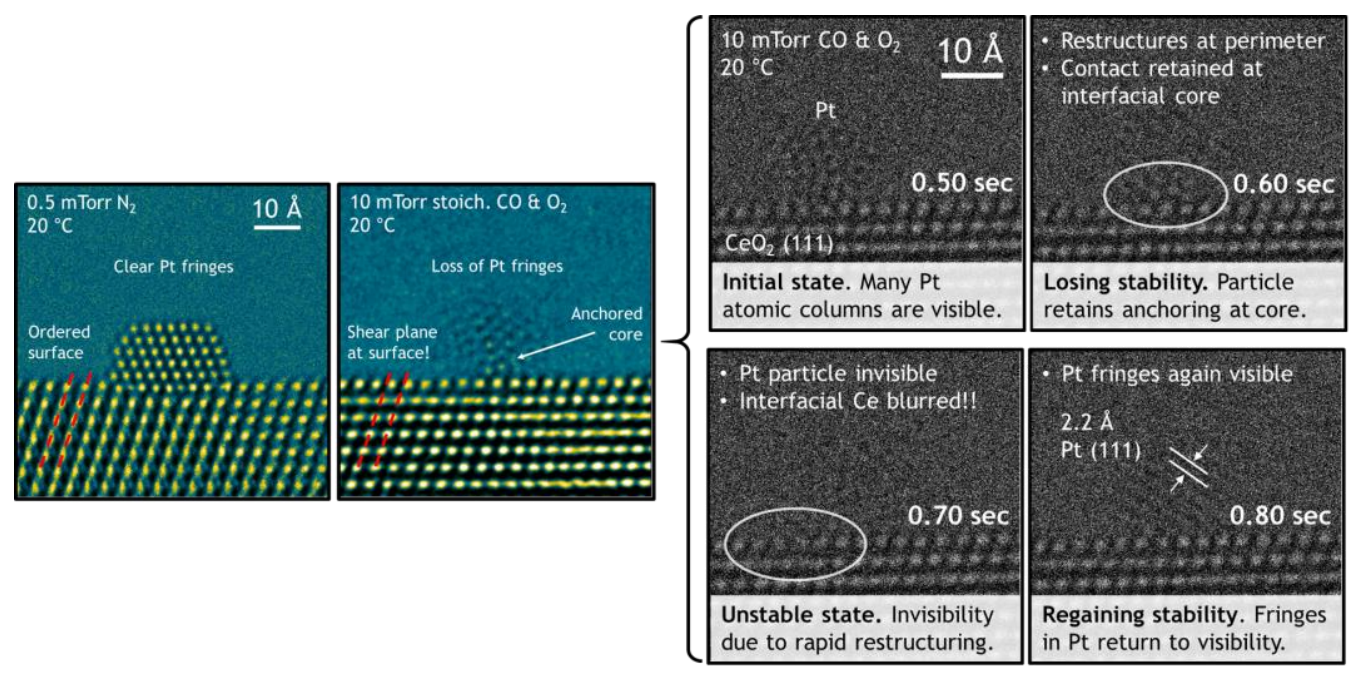

Figure 2. Time-resolved in situ TEM images of $\mathrm{Pt} \mathrm{NP}$ on (111) $\mathrm{CeO}_{2}$ surface is stable in $\mathrm{N}_{2}$ but dynamically restructuring in $\mathrm{CO}$ and $\mathrm{O}_{2}$. The core of the Pt appears to remain anchored. A shear plane (red lines) suggests a high concentration of oxygen vacancies in the $\mathrm{CeO}_{2}$. The time-resolved breakdown of the image-series reveals the atomic-scale dynamics that occur in reactant gases. Interestingly, interfacial Ce columns are blurred while the Pt undergoes its fastest reconfiguration, i.e., when it is least stable $(\mathrm{t}=$ 0.70 seconds).

\section{References}

1. Cargnello, M., et al., Science, 2013, 341, 6147.

2. Vayssilov, G. N., et al., Nature Materials, 2011, 10, 310.

3. Miller, B., Crozier, P. A. Microscopy and Microanalysis, 2014, 20, 815.

4. Lawrence, E. L., et al., Microscopy and Microanalysis, 2018, 24(S1).

5. Gai, P. L., Phil. Mag. A, 1981, 43, 841.

6. We gratefully acknowledge NSF grant CBET-1604971 and the facilities at the National Institute of Standards and Technology at Gaithersburg as well as those at the John M. Cowley Center for High Resolution Electron Microscopy at Arizona State University. 